\title{
LOCAL MOVES AND RESTRICTIONS ON THE JONES POLYNOMIAL
}

\author{
SANDY GANZELL
}

\begin{abstract}
We analyze various local moves on knot diagrams to show that Jones polynomials must have certain algebraic properties. In particular, we show that the Jones polynomial of a knot cannot be a nontrivial monomial.
\end{abstract}

\section{INTRODUCTION}

A local move on a link diagram is the substitution of a given subdiagram for another, that results in another link diagram. Reidemeister moves are the standard examples of local moves, but a local move need not preserve the link type, or even the number of components of the link. A local move $M$ is called an unknotting move if repeated applications of $M$, together with Reidemeister moves, will unknot every knot. The simplest unknotting move is a crossing change; a standard exercise in undergraduate knot theory is to show that any diagram of a knot can be transformed into a diagram of the unknot by crossing changes. The $\Delta$-move, shown in figure 1 , is also an

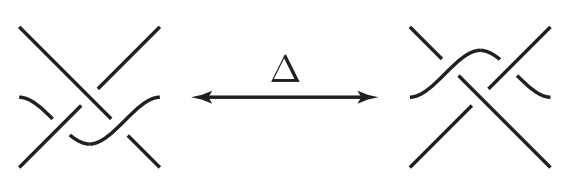

Figure 1 . The $\Delta$-move.

unknotting move [9], [10]. In section 2 we will examine the effect of the $\Delta$-move on the Jones polynomial.

We assume the reader is familiar with Kauffman's construction of the Jones polynomial ([7], [1], etc.). For the knot diagram $K$, the bracket polynomial is denoted $\langle K\rangle$, and the Jones polynomial is denoted $f_{K}(A)=$ $\left(-A^{3}\right)^{-w}\langle K\rangle$, where $w$ is the writhe of $K$. We use $A=t^{-1 / 4}$ for the indeterminate, and let $d=-A^{2}-A^{-2}$, so that $\langle\bigcirc K\rangle=d\langle K\rangle$. It is understood that "polynomial" is to mean Laurent polynomial, since $\langle K\rangle \in \mathbb{Z}\left[A, A^{-1}\right]$.

2010 Mathematics Subject Classification. 57M25, 57M27. 
As usual, the entire knot is not always drawn in the calculation of the bracket polynomial; two diagrams in a calculation should be presumed identical outside of the portion drawn. For example, part of the bracket calculation of a diagram containing the tangle $T$ might be written

$$
\langle\mathcal{T}\rangle=p_{1}\langle\widetilde{\sim}\rangle+p_{2}\langle)(\rangle \text {, }
$$

where $p_{1}$ and $p_{2}$ are polynomials in $A$. Thus if $K$ is the knot formed by closing the tangle $T$, we have $\langle K\rangle=p_{1} d+p_{2}$. Note that tangles in this paper will always be closed "above," meaning the strands on the left side will be connected to the corresponding strands on the right.

Similarly, the bracket calculation of a diagram containing a 3 -tangle could be written

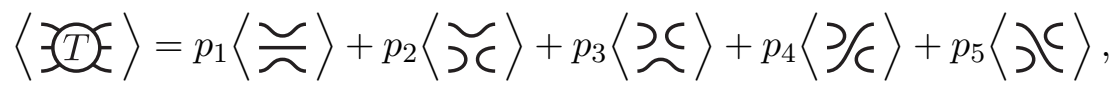

where $p_{1}, p_{2}, p_{3}, p_{4}, p_{5}$ are polynomials in $A$. If $K$ is the diagram formed by closing this 3 -tangle $T$, then $\langle K\rangle=p_{1} d^{2}+p_{2} d+p_{3} d+p_{4}+p_{5}$.

When we write the traditional Jones polynomial with indeterminate $t$, we use the notation $V(t)=V_{K}(t) \in \mathbb{Z}\left[t^{1 / 2}, t^{-1 / 2}\right]$.

\section{Local Moves and the Jones Polynomial}

We first look at the effect of a crossing change on the Jones polynomial. We consider two knots, $K$ and $K^{\prime}$ whose diagrams differ by a single crossing change, and we determine that the difference of their Jones polynomials must be divisible by $A^{12}-1$. Since every knot can be transformed into any other by crossing changes, we have that the difference of any two Jones polynomials (for knots) has this common factor.

Theorem 1. Let $K$ and $K^{\prime}$ be two knots. Then $f_{K}(A)-f_{K^{\prime}}(A)$ is divisible by $A^{12}-1$.

Proof. Any diagram with a crossing is equivalent ${ }^{1}$ to a diagram in figure 2. (To see this, take a ball containing the crossing, lift it up out of the paper and drag it to the far right of the diagram. Then move the NE and SE strands above the remainder of the diagram.)

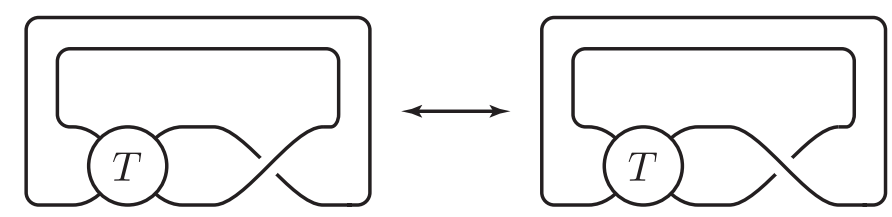

Figure 2. Two knots that differ by a crossing change.

\footnotetext{
${ }^{1}$ It is not necessary to draw the diagram this way; the calculations remain valid if we leave the crossing in place anywhere in the diagram. For virtual knots (section 3) the strands may need to be connected above and below.
} 
Let $K$ be the diagram on the left of figure 2 , and $K^{\prime}$ be the diagram on the right. Take the writhe of $K$ to be $w$, so the writhe of $K^{\prime}$ will be $w \pm 2$. We compute $f_{K}(A)-f_{K^{\prime}}(A)$. Suppose that

$$
\langle\widetilde{T}\rangle=p_{1}\langle\widetilde{\sim}\rangle+p_{2}\langle)(\rangle
$$

where $p_{1}$ and $p_{2}$ are polynomials in $A$. Then

$$
\begin{aligned}
& \left\langle T 2\langle\rangle=A\langle T\rangle(\rangle+A^{-1}\langle T(T)\rangle\right. \\
& =A p_{1}\langle)(\rangle+A p_{2} d\langle)(\rangle+A^{-1} p_{1}\langle\tilde{\sim}\rangle+A^{-1} p_{2}\langle)(\rangle . \\
& \langle K\rangle=A p_{1}+A p_{2} d+A^{-1} p_{1} d+A^{-1} p_{2} \\
& =p_{1}\left(A+A^{-1} d\right)+p_{2}\left(A d+A^{-1}\right) \\
& =p_{1}\left(-A^{-3}\right)+p_{2}\left(-A^{3}\right) \text {. }
\end{aligned}
$$

Thus $f_{K}(A)=\left(-A^{3}\right)^{-w}\left[p_{1}\left(-A^{-3}\right)+p_{2}\left(-A^{3}\right)\right]$.

Similarly,

$$
\begin{aligned}
\langle\text { TT }\rangle\rangle & =A\langle\text { TT }\rangle+A^{-1}\langle\text { TT } C . \\
\left\langle K^{\prime}\right\rangle & =A p_{1} d+A p_{2}+A^{-1} p_{1}+A^{-1} p_{2} d \\
& =p_{1}\left(A d+A^{-1}\right)+p_{2}\left(A+A^{-1} d\right) \\
& =p_{1}\left(-A^{3}\right)+p_{2}\left(-A^{-3}\right) .
\end{aligned}
$$

Thus $f_{K^{\prime}}(A)=\left(-A^{3}\right)^{-w \pm 2}\left[p_{1}\left(-A^{3}\right)+p_{2}\left(-A^{-3}\right)\right]$

$$
=\left(-A^{3}\right)^{-w}\left(A^{ \pm 6}\right)\left[p_{1}\left(-A^{3}\right)+p_{2}\left(-A^{-3}\right)\right] .
$$

So either

$$
\begin{aligned}
f_{K}(A)-f_{K^{\prime}}(A) & =\left(-A^{3}\right)^{-w} p_{2}\left(-A^{3}+A^{-9}\right) \\
& =\left(-A^{3}\right)^{-w+3} p_{2}\left(A^{12}-1\right),
\end{aligned}
$$

or

$$
\begin{aligned}
f_{K}(A)-f_{K^{\prime}}(A) & =\left(-A^{3}\right)^{-w} p_{1}\left(-A^{-3}+A^{9}\right) \\
& =\left(-A^{3}\right)^{-w-1} p_{1}\left(A^{12}-1\right) .
\end{aligned}
$$

One might hope to use this technique to refute Nakanishi's Conjecture that the 4-move (figure 3 ) is an unknotting move. Indeed, we can show that

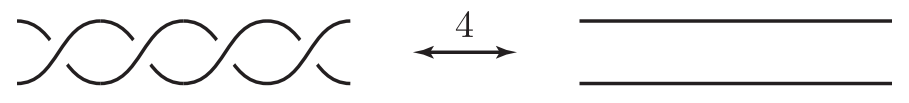

Figure 3. The 4-move.

for knots that differ by 4-moves, the difference of their Jones polynomials must be divisible by a polynomial $p(A)$. So we could test a potential counterexample $K$ by computing $f_{K}(A)-1$ and determining whether $p(A)$ was 
a factor. But the polynomial $p(A)$ turns out to be $A^{12}-1$, the same as for a crossing change. So every knot will pass this test.

We now return to the $\Delta$-move (figure 1), and determine its effect on the Jones polynomial.

Theorem 2. Let $K$ and $K^{\prime}$ be two knots. Then $f_{K}(A)-f_{K^{\prime}}(A)$ is divisible by $A^{16}-A^{12}-A^{4}+1$.

Proof. We consider knots $K$ and $K^{\prime}$ that differ by a $\Delta$-move, as in figure 4 . We will show that $f_{K}(A)-f_{K^{\prime}}(A)$ is divisible by $A^{16}-A^{12}-A^{4}+1$. Since $\Delta$ is an unknotting move, the theorem follows.

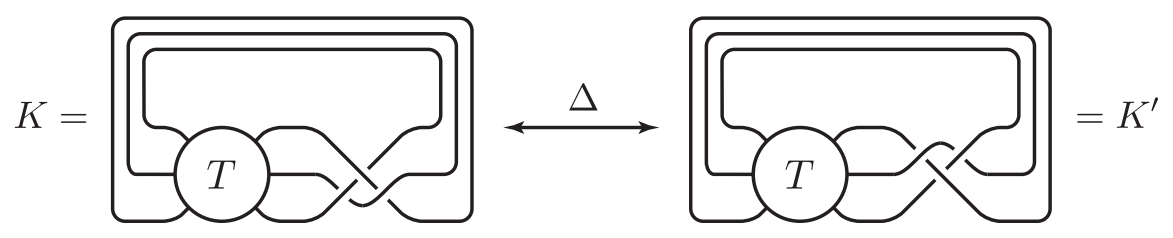

FIGURE 4

We calculate

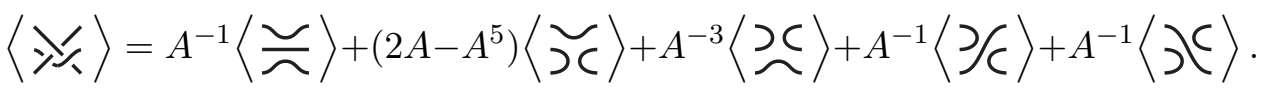

Now suppose

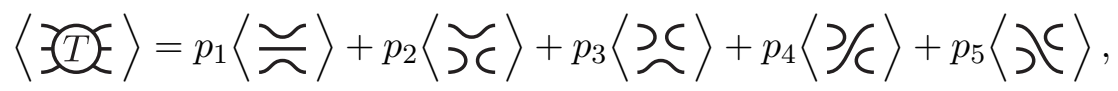

where $p_{1}, p_{2}, p_{3}, p_{4}, p_{5}$ are polynomials in $A$. Then

$$
\begin{aligned}
\langle K\rangle= & p_{1}\left[A^{-1} d^{2}+\left(2 A-A^{5}\right) d+A^{-3} d+A^{-1}+A^{-1}\right] \\
& +p_{2}\left[A^{-1} d+\left(2 A-A^{5}\right) d^{2}+A^{-3}+A^{-1} d+A^{-1} d\right] \\
& +p_{3}\left[A^{-1} d+\left(2 A-A^{5}\right)+A^{-3} d^{2}+A^{-1} d+A^{-1} d\right] \\
& +p_{4}\left[A^{-1}+\left(2 A-A^{5}\right) d+A^{-3} d+A^{-1}+A^{-1} d^{2}\right] \\
& +p_{5}\left[A^{-1}+\left(2 A-A^{5}\right) d+A^{-3} d+A^{-1} d^{2}+A^{-1}\right] \\
= & p_{1}\left(A^{7}+A^{-1}\right)+p_{2}\left(-A^{9}\right)+p_{3}\left(-A^{5}-A^{-3}+A^{-7}\right) \\
& +p_{4}\left(A^{7}+A^{-1}\right)+p_{5}\left(A^{7}+A^{-1}\right) .
\end{aligned}
$$

Similarly,

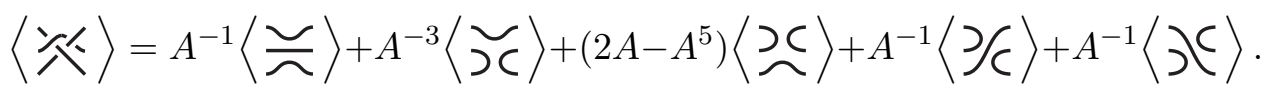


Thus

$$
\begin{aligned}
\left\langle K^{\prime}\right\rangle= & p_{1}\left[A^{-1} d^{2}+\left(2 A-A^{5}\right) d+A^{-3} d+A^{-1}+A^{-1}\right] \\
& +p_{2}\left[A^{-1} d+\left(2 A-A^{5}\right)+A^{-3} d^{2}+A^{-1} d+A^{-1} d\right] \\
& +p_{3}\left[A^{-1} d+\left(2 A-A^{5}\right) d^{2}+A^{-3}+A^{-1} d+A^{-1} d\right] \\
& +p_{4}\left[A^{-1}+\left(2 A-A^{5}\right) d+A^{-3} d+A^{-1}+A^{-1} d^{2}\right] \\
& +p_{4}\left[A^{-1}+\left(2 A-A^{5}\right) d+A^{-3} d+A^{-1} d^{2}+A^{-1}\right] \\
= & p_{1}\left(A^{7}+A^{-1}\right)+p_{2}\left(-A^{5}-A^{-3}+A^{-7}\right)+p_{3}\left(-A^{9}\right) \\
& +p_{4}\left(A^{7}+A^{-1}\right)+p_{5}\left(A^{7}+A^{-1}\right) .
\end{aligned}
$$

Since the writhe of $K$ equals the writhe of $K^{\prime}$, we have

$$
\begin{aligned}
& f_{K}(A)-f_{K^{\prime}}(A)=\left(-A^{3}\right)^{-w} {\left[p_{2}\left(-A^{9}+A^{5}+A^{-3}-A^{-7}\right)\right.} \\
&\left.-p_{3}\left(-A^{9}+A^{5}+A^{-3}-A^{-7}\right)\right] \\
&=(-A)^{-3 w-7}\left(p_{2}-p_{3}\right)\left(A^{16}-A^{12}-A^{4}+1\right) .
\end{aligned}
$$

Corollary 3. The Jones polynomial of a knot cannot have the form $r A^{n}$ unless $n=0$ and $r=1$.

Proof. Suppose $f_{K}(A)$ is the Jones polynomial of the knot $K$. Since the Jones polynomial of the unknot equals 1 , theorem 2 tells us $f_{K}(A)-1$ is divisible by $A^{16}-A^{12}-A^{4}+1$. But $(A-1)^{2}$ is a factor of $A^{16}-A^{12}-A^{4}+1$, hence $f_{K}(A)-1$ has a double root at $A=1$. But $r A^{n}-1$ has no double roots unless $r A^{n}-1 \equiv 0$.

Note that $J(A)=A^{16}-A^{12}-A^{4}+1$ is precisely the difference of the Jones polynomials of the unknot and the left-handed trefoil. Thus $J(A)$ is maximal in the sense that any polynomial that divides the difference of every pair of Jones polynomials will divide $J(A)$.

Switching to the traditional notation for the Jones polynomial, we can use theorem 2 to reproduce some previously known [12], [14] restrictions on $V(t)$. Define

$$
v_{n}=\left.\frac{1}{n !} \frac{d^{n}}{d t^{n}} V(t)\right|_{t=1} .
$$

Then as seen in [2], $v_{n}$ is a $\mathbb{Z}$-valued Vassiliev invariant of degree at most $n$.

Corollary 4. If $V(t)$ is the Jones polynomial of a knot, then

(1) $V(1)=1$.

(2) $v_{1}=0$

(3) $3 \mid v_{2}$.

(4) $3 \mid v_{3}$.

Proof. Theorem 2 implies $V(t)-1$ is a multiple of $s(t)=t^{4}-t^{3}-t+1$, so we can write $V(t)=q(t) s(t)+1$. Since $s(1)=0$, part (1) follows immediately. Now, $V^{\prime}(t)=q^{\prime}(t) s(t)+q(t) s^{\prime}(t)$. Since $s(1)=s^{\prime}(1)=0$, part (2) follows. 
Similarly, we calculate $v_{2}=3 q(1)$ and $v_{3}=3 q(1)+3 q^{\prime}(1)$, proving parts (3) and (4).

\section{Virtual Knots and Forbidden Moves}

Equivalence of virtual knots may be defined by means of a set of local moves (the extended Reidemeister moves) on their diagrams. Figure 5 illustrates the extended Reidemeister moves: the classical Reidemeister moves $R 1, R 2, R 3$, the virtual moves $V 1, V 2, V 3$ and the semivirtual move $S V$. We may then define a virtual knot to be an equivalence class of virtual
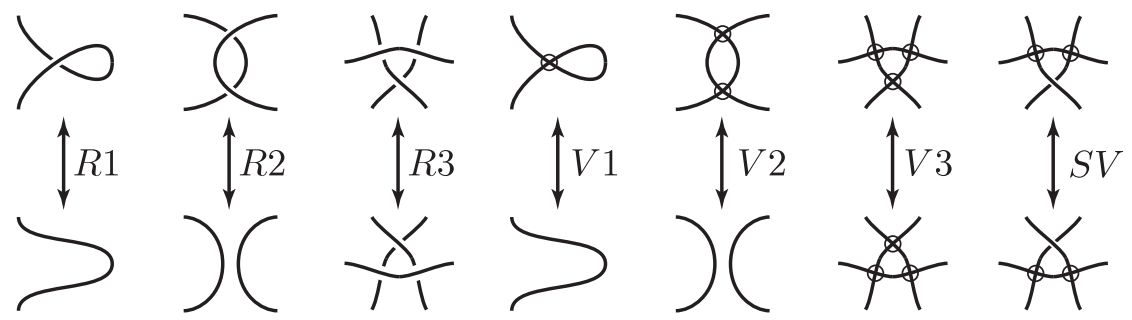

FiguRE 5. The extended Reidemeister moves.

diagrams modulo these moves.

In [4] it is proved that two classical knots $K_{1}$ and $K_{2}$ are equivalent under extended Reidemeister moves if and only if they are equivalent under classical Reidemeister moves. Thus virtual knot theory may be considered a generalization of the classical theory. The Jones polynomial is computed for virtual knots through the bracket polynomial exactly as it is for classical knots by simply ignoring the virtual crossings [8].

Theorems 1 and 2 fail for virtual knots for two reasons: first, crossing changes and $\Delta$-moves are not unknotting moves for virtual knots; and second, the bracket polynomial for virtual tangles cannot be written as in equation 1 (from the introduction). We must write

$$
\langle\widetilde{T}\rangle\rangle=p_{1}\langle\tilde{\sim}\rangle+p_{2}\langle)(\rangle+p_{3}\langle\chi\rangle \text {. }
$$

To prove the analogous theorems for virtual knots, we consider an unknotting set of moves, namely the forbidden moves (figure 6 ). The forbidden over

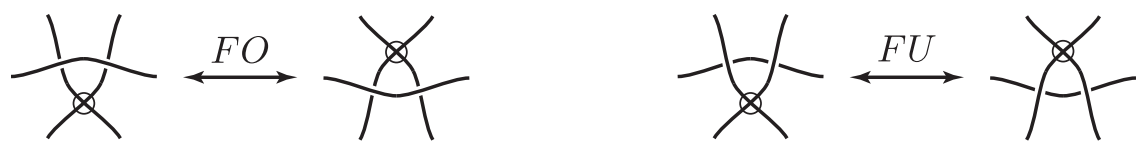

FiguRE 6. Forbidden moves.

move $F O$ moves a strand of the diagram "over" a virtual crossing, while the forbidden under move $F U$ moves a strand "under" a virtual crossing. Neither of these moves can be obtained as a sequence of extended Reidemeister 
moves: If we allow one forbidden move but not the other, we obtain what are known as welded knots, developed by Satoh in [13] and Kamada in [5]. If we allow both forbidden moves, then any virtual knot can be transformed into any other virtual knot [6], [11], [3].

The method is the same as for theorem 2. Here we observe that for a virtual knot $K$ containing a virtual 3 -tangle $T$, we can write

$$
\langle\bar{T} \bar{T}\rangle=\sum_{i=1}^{15} p_{i}\left\langle F_{i}\right\rangle \text {, }
$$

where the $F_{i}$ are the 15 basic virtual 3 -tangles (i.e., those without classical crossings and disjoint unknots), namely,

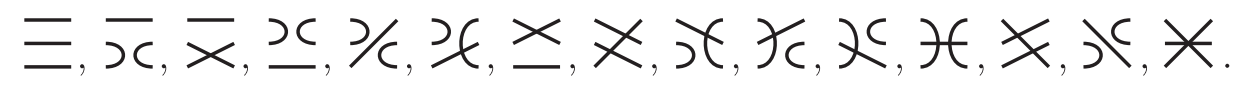

(All crossings in these 15 tangles should be taken as virtual crossings.) We then consider two virtual knots, $K$ and $K^{\prime}$, that differ by a $F U$-move, and compute

$$
\langle K\rangle-\left\langle K^{\prime}\right\rangle=\left(A^{-4} p_{6}-A^{-6} p_{9}-A^{-4} p_{10}+A^{-6} p_{11}\right)\left(A^{10}-A^{6}-A^{4}+1\right) .
$$

Since the two knots have the same writhe, the difference of Jones polynomials is a multiple of $A^{10}-A^{6}-A^{4}+1$. The calculation is similar for two knots that differ by a $F O$-move. The difference of Jones polynomials is again a multiple of $A^{10}-A^{6}-A^{4}+1$. Hence we have the theorem analogous to theorem 2 for virtual knots.

Theorem 5. Let $K$ and $K^{\prime}$ be two virtual knots. Then $f_{K}(A)-f_{K^{\prime}}(A)$ is divisible by $A^{10}-A^{6}-A^{4}+1$.

Since $A^{10}-A^{6}-A^{4}+1$ is a multiple of $(A-1)^{2}$, corollary 3 holds for virtual knots as well.

Corollary 6. The Jones polynomial of a virtual knot cannot have the form $r A^{n}$ unless $n=0$ and $r=1$.

Note that $J_{V}(A)=A^{10}-A^{6}-A^{4}+1$ is the difference of the Jones polynomials of the unknot and the left-handed virtual trefoil (figure 7 ). Thus $J_{V}(A)$ is maximal in the sense that any polynomial that divides the difference of every pair of Jones polynomials will divide $J_{V}(A)$.

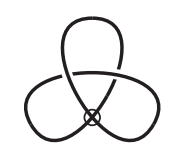

Figure 7. Virtual trefoil. 


\section{REFERENCES}

[1] C. C. Adams, The Knot Book, An Elementary Introduction to the Mathematical Theory of Knots. American Mathematical Society, 2004.

[2] J. Birman and X. S. Lin, Knot polynomials and Vassiliev's invariants. Invent. Math. 111:225-270, 1993.

[3] A. Crans, S. Ganzell and B. Mellor, The Forbidden Number of a Knot. arXiv:1305.5200v1 [math.GT], 2013.

[4] M. Goussarov, M. Polyak, and O. Viro, Finite-type invariants of classical and virtual knots. Topology, 39:1045-1068, 2000.

[5] S. Kamada, Braid presentation of virtual knots and welded knots. Osaka J. Math., 44, No. 2:441-458, 2007.

[6] T. Kanenobu, Forbidden moves unknot a virtual knot. J. Knot Theory Ramifications, 10:89-96, 2001.

[7] L. H. Kauffman, New Invariants in the Theory of Knots. Amer. Math. Mon., 195-242, March, 1988.

[8] L. H. Kauffman, Virtual knot theory. Europ. J. Combinatorics, 20:663-691, 1999.

[9] S. V. Matveev, Generalized surgeries of three-dimensional manifolds and representations of homology sphere, Mat. Zametki 42, 268-278, 345, 1987, in Russian. English translation: Math. Notes 42, 651-656, 1987.

[10] H. Murakami and Y. Nakanishi, On a certain move generating link-homology. Math. Ann., 284:75-89, 1989.

[11] S. Nelson, Unknotting virtual knots with Gauss diagram forbidden moves. J. Knot Theory Ramifications, 10:931-935, 2001.

[12] M. Polyak and O. Viro, Gauss diagram formulas for Vassiliev invariants. Int. Math. Res. Notes, 11:445-454, 1994.

[13] S. Satoh, Virtual knot presentation of ribbon torus-knots. J. Knot Theory Ramifications, 9:531-542, 2000.

[14] A. Stoimenow, On some restrictions to the values of the Jones polynomial, Indiana Univ. Math. J. 54 (2) (2005), 557574.

Department of Mathematics and Computer Science, St. Mary's College of Maryland, St. Mary's City, MD 20686

E-mail address: sganzell@smcm.edu 